\title{
OBSERVER UNTUK SISTEM KONTROL LINIER KONTINU
}

\author{
SUKMA HAYATI, ZULAKMAL \\ Program Studi Matematika, \\ Fakultas Matematika dan Ilmu Pengetahuan Alam, Universitas Andalas, \\ Kampus UNAND Limau Manis Padang, Indonesia, \\ email : nyuma_kamiya@yahoo.com
}

\begin{abstract}
Abstrak. Dalam makalah ini dikaji syarat agar diperoleh persamaan observer untuk suatu sistem kontrol linier kontinu. Estimasi yang baik memenuhi $\overline{\mathbf{e}}(t) \rightarrow \mathbf{0}$ bila $t \rightarrow \infty$, atau stabil asimtotik. Kestabilan asimtotik sistem $\dot{\overline{\mathbf{e}}}$ diperoleh apabila bagian riil dari semua nilai eigen matriks $(A-L C)$ bernilai negatif. Bentuk eksplisit matriks $L$ dapat diperoleh apabila memenuhi syarat tertentu yang dipaparkan pada paper ini.
\end{abstract}

Kata Kunci: Observer, estimasi error, stabil asimtotik

\section{Pendahuluan}

Diberikan suatu kontrol linier sebagai berikut :

$$
\begin{aligned}
\dot{\mathbf{x}}(t) & =A \mathbf{x}(t)+B u(t), \\
y(t) & =C \mathbf{x}(t)
\end{aligned}
$$

dimana $A \in \mathbb{R}^{n \times n}, B \in \mathbb{R}^{n \times 1}$, dan $C \in \mathbb{R}^{1 \times n}$. Pada sistem (1.1), $\mathbf{x}(t) \in \mathbb{R}^{n}$ menyatakan vektor keadaan (state), $u(t) \in \mathbb{R}$ menyatakan input (control), dan $y \in \mathbb{R}$ menyatakan output.

Suatu observer untuk sistem (1.1) didefinisikan sebagai persamaan berikut:

$$
\dot{\widehat{\mathbf{x}}}(t)=A \widehat{\mathbf{x}}(t)+B u(t)+L(y(t)-\widehat{y}(t)),
$$

untuk suatu matriks $L \in \mathbb{R}^{n}$ dimana $\widehat{\mathbf{x}}(t) \in \mathbb{R}^{n}, \widehat{y}(t)=C \widehat{\mathbf{x}}(t)$.

Vektor $\widehat{\mathbf{x}}(t)$ memiliki peran sebagai estimator untuk $\mathbf{x}(t)$, dengan estimasi error $\overline{\mathbf{e}}(t)=\mathbf{x}(t)-\widehat{\mathbf{x}}(t)$. Estimasi yang baik mestilah memenuhi $\overline{\mathbf{e}}(t) \rightarrow \mathbf{0}$ bila $t \rightarrow \infty$, atau $\widehat{\mathbf{x}}(t) \rightarrow \mathbf{x}(t)$ bila $t \rightarrow \infty$. Dalam makalah ini dikaji syarat yang menjamin agar persamaan (1.2) merupakan suatu observer yang baik untuk sistem (1.1).

\section{Observer untuk Sistem Kontrol Linier Kontinu}

Persamaan (1.2) merupakan observer yang baik untuk sistem kontrol linier (1.1) apabila

$$
\overline{\mathbf{e}}=\mathbf{x}-\widehat{\mathbf{x}} \rightarrow \overline{\mathbf{0}}
$$


untuk $t \rightarrow \infty$. Dari (2.1) diperoleh

$$
\begin{aligned}
\dot{\overline{\mathbf{e}}} & =\dot{\mathbf{x}}-\dot{\widehat{\mathbf{x}}} \\
& =(A-L C) \overline{\mathbf{e}} .
\end{aligned}
$$

Jelas bahwa (2.2) merupakan suatu sistem persamaan diferensial linier orde 1 yang kestabilan asimtotiknya ditentukan oleh matriks $A-L C$. Bila sistem $\dot{\overline{\mathbf{e}}}(t)=(A-$ $L C) \overline{\mathbf{e}}(t)$ stabil asimtotik, yaitu bagian riil dari semua nilai eigen $(A-L C)$ adalah negatif, maka $\overline{\mathbf{e}}(t) \rightarrow \mathbf{0}$ untuk $t \rightarrow \infty$, sehingga $\dot{\widehat{\mathbf{x}}}(t)$ merupakan observer yang baik untuk $\dot{\mathbf{x}}(t)$. Dengan demikian diperlukan informasi tentang bentuk eksplisit dari matriks $L$ sedemikian sehingga bagian riil dari semua nilai eigen matriks $(A-L C)$ adalah negatif.

Bentuk eksplisit $L$ dapat dicari dengan menggunakan bentuk dual dari (1.1) yaitu

$$
\dot{\overline{\mathbf{e}}}=A^{T} \overline{\mathbf{e}}+C^{T} u \text {. }
$$

Jika $u=-L^{T} \overline{\mathbf{e}}$, maka (2.3) dapat ditulis menjadi

$$
\dot{\overline{\mathbf{e}}}=\left(A^{T}-C^{T} L^{T}\right) \overline{\mathbf{e}}
$$

yang solusinya adalah

$$
\overline{\mathbf{e}}(t)=e^{\left(A^{T}-C^{T} L^{T}\right) t} \overline{\mathbf{e}}(0) .
$$

Dari (2.5) terlihat bahwa $\overline{\mathbf{e}}(t) \rightarrow \mathbf{0}$ jika bagian riil dari semua nilai eigen matriks $\left(A^{T}-C^{T} L^{T}\right)$ adalah negatif.

Teorema berikut menginformasikan syarat yang menjamin eksistensi matriks $L$ sedemikian sehingga nilai eigen $\left(A^{T}-C^{T} L^{T}\right)$ dapat dibuatkan sesuai keinginan.

Teorema 2.1. [2] Jika $\left(A^{T}, C^{T}\right)$ adalah terkontrol lengkap maka terdapat matriks $L \in \mathbb{R}^{n \times 1}$ sedemikian sehingga nilai eigen dari matriks $\left(A^{T}-C^{T} L^{T}\right)$ dapat ditempatkan secara sebarang.

Bukti. Sistem $\left(A^{T}, C^{T}\right)$ diubah menjadi bentuk kanonik terkontrol menggunakan transformasi $Q$, yaitu

$$
Q=M_{\mathcal{C}} W
$$

dimana $M_{\mathcal{C}}$ adalah matriks keterkontrolan

$$
\begin{gathered}
M_{\mathcal{C}}=\left[C^{T} A^{T} C^{T} \cdots\left(A^{T}\right)^{n-1} C^{T},\right] \\
W=\left[\begin{array}{ccccc}
a_{n-1} & a_{n-2} & \cdots & a_{1} & 1 \\
a_{n-2} & a_{n-3} & \cdots & 1 & 0 \\
\vdots & \vdots & \ddots & \vdots & \vdots \\
a_{1} & 1 & \cdots & 0 & 0 \\
1 & 0 & \cdots & 0 & 0
\end{array}\right]
\end{gathered}
$$

dan $a_{i}, i=1,2, \cdots, n$ adalah koefisien dari polinomial karakteristik

$$
\left|s I-A^{T}\right|=s^{n}+a_{1} s^{(n-1)}+\cdots+a_{n-1} s+a_{n} .
$$


Definisikan variabel keadaan baru $\widehat{\mathbf{e}}$ dengan

$$
\overline{\mathbf{e}}=Q \widehat{\mathbf{e}} .
$$

Jika $\operatorname{rank}\left(M_{\mathcal{C}}\right)$ adalah $n$ (berarti sistem terkontrol lengkap), maka matriks $Q$ memiliki invers. Sehingga persamaan sistem $\dot{\overline{\mathbf{e}}}=A^{T} \overline{\mathbf{e}}+C^{T} u$ menjadi

$$
\dot{\widehat{\mathbf{e}}}=Q^{-1} A^{T} Q \widehat{\mathbf{e}}+Q^{-1} C^{T} u
$$

dimana

$$
Q^{-1} A^{T} Q=\left[\begin{array}{ccccc}
0 & 1 & 0 & \cdots & 0 \\
0 & 0 & 1 & \cdots & 0 \\
\cdots & \cdots & \cdots & \ddots & \cdots \\
0 & 0 & 0 & \cdots & 1 \\
-a_{n} & -a_{n-1} & -a_{n-2} & \cdots & -a_{1}
\end{array}\right]
$$

dan

$$
Q^{-1} C^{T}=\left[\begin{array}{c}
0 \\
0 \\
\vdots \\
0 \\
1
\end{array}\right]
$$

Persamaan (2.7) dan (2.8) merupakan bentuk kanonik terkontrol. Kemudian akan dibuktikan jika sistem terkontrol lengkap, maka dimungkinkan untuk memilih nilai eigen yang diinginkan, misalkan $s=s_{1}, s=s_{2}, \cdots, s=s_{n}$. Persamaan karakteristiknya berubah menjadi

$$
\left(s-s_{1}\right)\left(s-s_{2}\right) \cdots\left(s-s_{n}\right)=s^{n}+\alpha_{1} s^{(n-1)}+\cdots+\alpha_{(n-1)} s+\alpha_{n}=0 .
$$

Tulis

$$
L^{T} Q=\left[\delta_{n} \delta_{n-1} \cdots \delta_{1}\right] .
$$

Bila $u=-L^{T} Q \widehat{\mathbf{e}}$ digunakan untuk mengontrol sistem (2.6), maka diperoleh persamaan

$$
\dot{\hat{\mathbf{e}}}=Q^{-1} A^{T} Q \widehat{\mathbf{e}}-Q^{-1} C^{T} L^{T} Q \widehat{\mathbf{e}} .
$$

Persamaan karakteristik dari (2.11) adalah

$$
\left|s I-Q^{-1} A^{T} Q+Q^{-1} C^{T} L^{T} Q\right|=0 .
$$

Persamaan karakteristik ini sama dengan persamaan karakteristik untuk sistem $\dot{\overline{\mathbf{e}}}=A^{T} \overline{\mathbf{e}}+C^{T} u$ dengan $u=-L^{T} \overline{\mathbf{e}}$ yang digunakan sebagai kontrol, yaitu

$$
\begin{aligned}
\dot{\overline{\mathbf{e}}} & =A^{T} \overline{\mathbf{e}}+C^{T} u \\
& =\left(A^{T}-C^{T} L^{T}\right) \overline{\mathbf{e}} .
\end{aligned}
$$

Persamaan karakteristik untuk sistem (2.13) adalah

$$
\begin{aligned}
\left|s I-A^{T}+C^{T} L^{T}\right| & =\left|Q^{-1}\left(s I-A^{T}+C^{T} L^{T}\right) Q\right| \\
& =\left|s I-Q^{-1} A^{T} Q+Q^{-1} C^{T} L^{T} Q\right| \\
& =0 .
\end{aligned}
$$


Substitusikan persamaan (2.7), (2.8), dan (2.10) ke dalam persamaan karakteristik (2.12) diperoleh

$$
\begin{aligned}
\left|s I-Q^{-1} A^{T} Q+Q^{-1} C^{T} L^{T} Q\right| & \\
& =\mid s I-\left[\begin{array}{ccccc}
0 & 1 & 0 & \cdots & 0 \\
0 & 0 & 1 & \cdots & 0 \\
\cdots & \cdots & \cdots & \ddots & \cdots \\
0 & 0 & 0 & \cdots & 1 \\
-a_{n} & -a_{n-1} & -a_{n-2} & \cdots & -a_{1}
\end{array}\right]+\left[\begin{array}{c}
0 \\
0 \\
\vdots \\
0 \\
1
\end{array}\right]\left[\delta_{n} \delta_{n-1} \cdots \delta_{1}\right] \\
& =\mid \begin{array}{cccc}
s & -1 & \cdots & 0 \\
0 & s & \cdots & 0 \\
\cdots & \cdots & \cdots & \ddots
\end{array} \\
& =s_{n}+\left(a_{1}+\delta_{1}\right) s^{(n-1)}+\cdots+\left(a_{n-1}+\delta_{n-1}\right) s+\left(a_{n}+\delta_{n}\right) \\
& =0
\end{aligned}
$$

Persamaan karakteristik ini sama dengan persamaan karakteristik (2.9). Sehingga dengan menyamakan koefisiennya diperoleh

$$
\begin{gathered}
a_{1}+\delta_{1}=\alpha_{1} \\
a_{2}+\delta_{2}=\alpha_{2} \\
\vdots \\
a_{n}+\delta_{n}=\alpha_{n} \\
\\
L^{T}=\left[\alpha_{n}-a_{n} \alpha_{n-1}-a_{n-1} \cdots \delta_{1}-a_{1}\right] Q^{-1} .
\end{gathered}
$$

Berikut ini akan dikemukakan langkah-langkah untuk mendapatkan matriks $L$. Misalkan nilai eigen yang diinginkan adalah $s=s_{1}, s=s_{2}, \cdots, s=s_{n}$. Persamaan karakteristik dari $A_{L^{T}}=A^{T}-C^{T} L^{T}$ adalah

$$
\begin{aligned}
\left|s I-A_{L}^{T}\right| & =\left(s-s_{1}\right)\left(s-s_{2}\right) \cdots\left(s-s_{n}\right) \\
& =s^{n}+\alpha_{1} s^{n-1}+\cdots+\alpha_{n-1} s+\alpha_{n} \\
& =0
\end{aligned}
$$

untuk suatu skalar $\alpha_{i}, i=1,2, \cdots, n$. Berdasarkan teorema Cayley-Hamilton untuk $A_{L^{T}}$, maka berlaku

$$
\phi\left(A_{L^{T}}\right)=A_{L^{T}}{ }^{n}+\alpha_{1} A_{L^{T}}^{n-1}+\cdots+\alpha_{n-1} A_{L^{T}}+\alpha_{n} I=0 .
$$


Perhatikan identitas berikut :

$$
\begin{aligned}
A_{L^{T}}= & \left(A^{T}\right)-C^{T} L^{T} \\
A_{L^{T}}{ }^{2}= & A_{L^{T}} A_{L^{T}} \\
= & \left(A^{T}\right)^{2}-A^{T}\left(C^{T}\right) L^{T}-\left(C^{T}\right) L^{T} A_{L^{T}} \\
& \vdots \\
A_{L^{T}}{ }^{n}= & A_{L^{T}}{ }^{n-1} A_{L^{T}} \\
= & \left(A^{T}\right)^{n}-\left(A^{T}\right)^{n-1}\left(C^{T}\right) L^{T}-\left(A^{T}\right)^{n-2}\left(C^{T}\right) L^{T} A_{L^{T}}-\left(A^{T}\right)^{n-3}\left(C^{T}\right) L^{T}\left(A_{L^{T}}\right)^{2} \\
& -\cdots-\left(A^{T}\right)\left(C^{T}\right) L^{T} A_{L^{T}}^{n-2}-\left(C^{T}\right) L^{T} A_{L^{T}}^{n-1}
\end{aligned}
$$

Substitusikan identitas tersebut ke persamaan (2.14), sehingga diperoleh persamaan berikut :

$$
\begin{aligned}
& \phi\left(A^{T}\right)=\left(C^{T}\right)\left(\alpha_{n-1} L^{T}+\alpha_{n-2} L^{T} A_{L^{T}}+\cdots+\alpha_{1} L^{T} A_{L^{T}}{ }^{n-2}-L^{T} A_{L^{T}}^{n-1}\right) \\
& +\left(A^{T}\right)\left(C^{T}\right)\left(\alpha_{n-2} L^{T}+\cdots+L^{T} A_{L^{T}}^{n-2}\right)+\cdots \\
& +\left(A^{T}\right)^{n-3}\left(C^{T}\right)\left(\alpha_{1} L^{T} A_{L^{T}}+\cdots+L^{T} A_{L^{T}}^{2}\right) \\
& \left(A^{T}\right)^{n-2}\left(C^{T}\right)\left(\alpha_{1} L^{T}+\cdots+L^{T} A_{L^{T}}\right)+\left(A^{T}\right)^{n-1}\left(C^{T}\right) L^{T} \\
& =\left[\left(C^{T}\right)\left(A^{T}\right)\left(C^{T}\right) \cdots\left(A^{T}\right)^{n-1}\left(C^{T}\right)\right] \\
& {\left[\begin{array}{c}
\alpha_{n-1} L^{T}+\alpha_{n-2} L^{T} A_{L^{T}}+\cdots+\alpha_{1} L^{T} A_{L^{T}}{ }^{n-2}-L^{T} A_{L^{T}}{ }^{n-1} \\
\alpha_{n-2} L^{T}+\cdots+L^{T} A_{L^{T}}{ }^{n-2} \\
\vdots \\
L^{T}
\end{array}\right]} \\
& \Leftrightarrow\left[\left(C^{T}\right)\left(A^{T}\right)\left(C^{T}\right) \cdots\left(A^{T}\right)^{n-1}\left(C^{T}\right)\right]^{-1} \phi\left(A^{T}\right)= \\
& {\left[\begin{array}{c}
\alpha_{n-1} L^{T}+\alpha_{n-2} L^{T} A_{L^{T}}+\cdots+\alpha_{1} L^{T} A_{L^{T}}{ }^{n-2}-L^{T} A_{L^{T}}{ }^{n-1} \\
\alpha_{n-2} L^{T}+\cdots+L^{T} A_{L^{T}}^{n-2} \\
\vdots \\
L^{T}
\end{array}\right]}
\end{aligned}
$$

Kemudian kalikan kedua sisi dengan $\left[\begin{array}{llll}0 & 0 & \cdots & 1\end{array}\right]$, diperoleh persamaan berikut:

$$
\begin{aligned}
& {\left[\begin{array}{llll}
0 & 0 & \cdots & 1
\end{array}\right]\left[\left(C^{T}\right)\left(A^{T}\right)\left(C^{T}\right) \cdots\left(A^{T}\right)^{n-1}\left(C^{T}\right)\right]^{-1} \phi\left(A^{T}\right) } \\
& =\left[\begin{array}{llll}
0 & 0 & \cdots & 1
\end{array}\right]\left[\begin{array}{c}
\alpha_{n-1} L^{T}+\alpha_{n-2} L^{T} A_{L^{T}}+\cdots+\alpha_{1} L^{T} A_{L^{T}}{ }^{n-2}-L^{T} A_{L^{T}}{ }^{n-1} \\
\alpha_{n-2} L^{T}+\cdots+L^{T} A_{L^{T}}{ }^{n-2} \\
\vdots \\
L^{T}
\end{array}\right] \\
= & L^{T}
\end{aligned}
$$

atau dapat ditulis sebagai

$$
L^{T}=\left[\begin{array}{lllll}
0 & 0 & \cdots & 0 & 1
\end{array}\right] M_{\mathcal{C}}^{-1} P_{c h, A_{L^{T}}}\left(A^{T}\right),
$$


dimana $M_{\mathcal{C}}$ adalah matriks keterkontrolan dan $P_{c h, A_{L^{T}}}\left(A^{T}\right)=\phi\left(A^{T}\right)$ adalah polinomial karakteristik untuk matriks $A_{L^{T}}$. Dengan demikian diperoleh

$$
L=\left(L^{T}\right)^{T}=P_{c h, A_{L^{T}}}^{T}\left(A^{T}\right)\left(M_{\mathcal{C}}^{-1}\right)^{T}\left[\begin{array}{c}
0 \\
0 \\
\vdots \\
0 \\
1
\end{array}\right]
$$

Persamaan (2.16) menunjukkan bahwa terdapat matriks $L \in \mathbb{R}^{n \times 1}$, sehingga nilai eigen matriks $A^{T}-L^{T} C^{T}$ dapat ditempatkan secara sebarang. Dengan perkataan lain (1.2) merupakan observer yang baik untuk sistem (1.1).

\section{Kesimpulan}

Diberikan suatu kontrol linier sebagai berikut :

$$
\dot{\mathbf{x}}(t)=A \mathbf{x}(t)+B u(t), y(t)=C \mathbf{x}(t)
$$

dimana $A \in \mathbb{R}^{n \times n}, B \in \mathbb{R}^{n \times 1}$, dan $C \in \mathbb{R}^{1 \times n}$. Pada sistem $(3.1), \mathbf{x}(t) \in \mathbb{R}^{n}$ menyatakan vektor keadaan (state), $u(t) \in \mathbb{R}$ menyatakan input (control), dan $y \in \mathbb{R}$ menyatakan output.

Suatu observer untuk sistem (3.1) didefinisikan sebagai persamaan berikut :

$$
\dot{\widehat{\mathbf{x}}}(t)=A \widehat{\mathbf{x}}(t)+B u(t)+L(y(t)-\widehat{y}(t)),
$$

untuk suatu matriks $L \in \mathbb{R}^{n}$ dimana $\widehat{\mathbf{x}}(t) \in \mathbb{R}^{n}, \widehat{y}(t)=C \widehat{\mathbf{x}}(t)$.

Error antara $\mathbf{x}(t)$ dengan estimator $\widehat{\mathbf{x}}(t)$ dinamakan error estimasi $\overline{\mathbf{e}}(t)$, yaitu $\overline{\mathbf{e}}(t)=\mathbf{x}-\widehat{\mathbf{x}}$, yang dibangun dari persamaan error dinamik $\dot{\overline{\mathbf{e}}}(t)=\dot{\mathbf{x}}(t)-\dot{\widehat{\mathbf{x}}}(t)$. Apabila $\dot{\overline{\mathbf{e}}}(t)=(A-L C) \overline{\mathbf{e}}(t)$ stabil asimtotik, yaitu bagian riil nilai eigen $(A-L C)<$ 0 , maka $\overline{\mathbf{e}}(t) \rightarrow \mathbf{0}$ untuk $t \rightarrow \infty$, dan $\dot{\widehat{\mathbf{x}}}(t)$ dikatakan observer yang baik untuk $\dot{\mathbf{x}}(t)$.

Bagian riil nilai eigen $(A-L C)$ dapat ditempatkan sebarang, yaitu apabila terdapat matriks $L \in \mathbb{R}^{n \times 1}$ sebagai berikut.

$$
L=\left(L^{T}\right)^{T}=P_{c h, A_{L} T}^{T}\left(A^{T}\right)\left(M_{\mathcal{C}}^{-1}\right)^{T}\left[\begin{array}{c}
0 \\
0 \\
\vdots \\
0 \\
1
\end{array}\right] .
$$

\section{Daftar Pustaka}

[1] Anton, H dan Rorres, C. 2004. Aljabar Linier Elementer. Edisi Kedelapan. Jilid 1. Penerbit Erlangga, Jakarta

[2] Antsaklis, P. J dan Michel, A. N. 2007. A Linear Systems Primer. Birkhauser, Boston

[3] Hendricks. E, Ole. J dan P. H. Soronsen. 2008. Linear System Control. Springer, Berlin 
102 Sukma Hayati, Zulakmal

[4] Ogata, K. 2002. Modern Control Engineering, Fourth Edition. Prentice-Hall, New Jersey

[5] Bastian. G dan N. Dautrebande. 1999. Positive linier Observers for Positive Linear Systems. Proceedings European Control Conference ECC'99, Paper F371

[6] Luenberger, B.G. 1979. Introduction to Dynamics System. John Wiley and Sons, California

[7] Golan, J.S. 2007. The Linear Algebra A Beginning Graduate Student Ought to Know. Second Edition. Springer, Dordrecht 